\title{
Les photographes ambulants
}

Conditions et pratiques professionnelles d'un métier itinérant, des années 1880 aux années 1930

Ilsen About

\section{(2) OpenEdition}

\section{Journals}

Édition électronique

URL : https://journals.openedition.org/tc/7611

DOI : 10.4000/tc. 7611

ISBN : 0248-6016

ISSN : 1952-420X

Éditeur

Éditions de l'EHESS

\section{Édition imprimée}

Date de publication : 24 décembre 2015

Pagination : 240

ISBN : 978-2-7132-2505-5

ISSN : 0248-6016

Référence électronique

IIsen About, «Les photographes ambulants », Techniques \& Culture [En ligne], 64 | 2015, mis en ligne le 24 mars 2016, consulté le 29 septembre 2022. URL : http://journals.openedition.org/tc/7611 ; DOI : https://doi.org/10.4000/tc.7611

Ce document a été généré automatiquement le 29 septembre 2022

Tous droits réservés 


\title{
Les photographes ambulants
}

\author{
Conditions et pratiques professionnelles d'un métier itinérant, des
} années 1880 aux années 1930

Ilsen About

\section{NOTE DE L'ÉDITEUR}

Nous vous invitons à consulter la synthèse de cet article (édition papier) en téléchargeant le document placé en annexe.

L'auteur remercie, pour leurs conseils éclairés, Clément Chéroux, Cosimo Chiarelli et Emmanuelle Fructus.

\section{Préambule}

Dans les années 1970, l'écrivain Evon Neal et la photographe Ann Parker entreprennent un voyage de plusieurs mois à travers le Guatemala à la recherche de photographes ambulants en activité. Ils rapportent une connaissance précise des pratiques quotidiennes exercées par ces opérateurs ainsi que des images qui rendent compte de la vitalité de cette activité et de sa fonction. À la fois ethnographes et anthropologues d'un groupe social, observateurs d'un métier et d'une pratique d'image, ils découvrent le rôle des photographes ambulants comme ciment social et constatent les multiples usages, dans la sphère privée ou publique, des tirages emportés par les passants. Ils rendent compte aussi de cet espace fragile - la tente du photographe - envisagé comme lieu d'une construction collective de l'image de soi qui s'élabore au moment des grandes foires dans les villages du pays, lorsque des dizaines de studios improvisés s'installent les uns à côté des autres. Mais leur livre, publié en 1982, ne montre pas seulement la survivance tardive d'un phénomène largement disparu ailleurs dans le monde, il donne à voir aussi la position essentielle, universelle et presque intemporelle des photographes mobiles dans la diffusion des pratiques sociales de l'image et dans l'essor de la culture visuelle sur la très longue durée (Neal \& Parker 1982). 
1 Invitation à un voyage dans le temps, l'ouvrage de Neal et Parker montre les visages et accorde une dignité à ces fabricants d'images dont la place dans l'histoire de la photographie reste particulièrement modeste malgré les quelques hommages qui leur ont été rendus (Charbonnier \& Soupault 1957 ; De Freitas \& Ghnassia 2001). Situés à distance des grands studios sédentaires de portraitistes, des laboratoires scientifiques et des ateliers d'artistes, ces artisans photographes occupent une position marginale, à la fois professionnelle et esthétique, dans l'histoire des régimes de production visuelle. Pourtant, leur rôle s'avère essentiel dans l'acculturation des sociétés contemporaines aux pratiques quotidiennes de l'image photographique et ils paraissent avoir contribué à une diffusion lente et durable des objets visuels dans la culture matérielle (fig. 1).

\section{Les petits métiers de Paris}

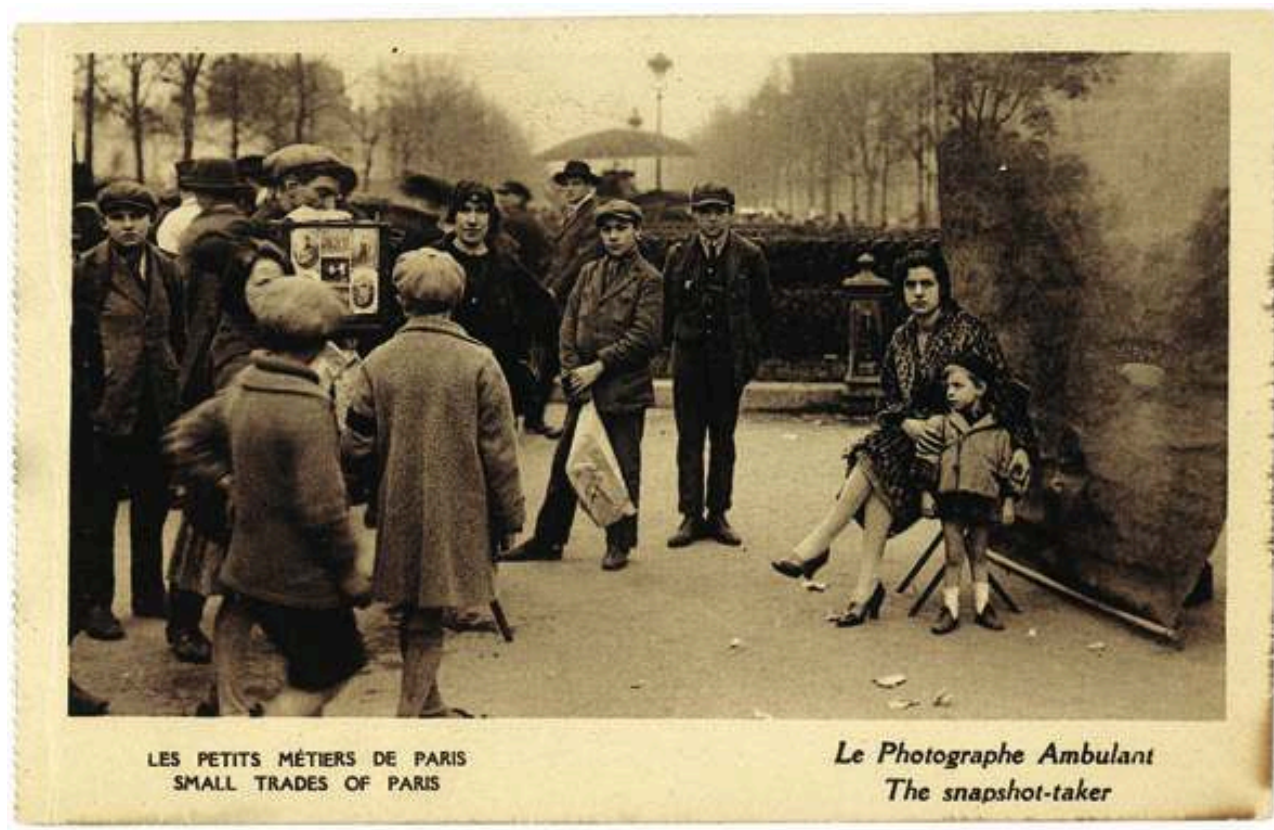

Le photographe ambulant/Small Trades of Paris. The snapshot-taker, carte postale, 9x13, ca. 1920, Collection particulière.

Auteur inconnu

\section{Des photographes voyageurs aux photographes ambulants}

Les études éparses consacrées à la photographie pratiquée par des ambulants et des forains abordent plusieurs aspects de cette profession, analysée essentiellement sous l'angle d'une pratique décalée, insolite, marginale ${ }^{1}$. Plusieurs recherches se sont consacrées aux aspects techniques de cette production, en particulier, pour la période ancienne, la ferrotypie et l'essor de la photographie imprimée sur cartes postales, dites carte photo (Armand 2011 ; Baudrain 2013). C'est surtout la pratique des photographes installés dans les fêtes foraines qui a focalisé l'attention ainsi que les relations de cette esthétique singulière avec les avant-gardes. La position spécifique de la photographie dans les foires en plein essor durant l'entre-deux-guerres, lieux caractéristiques du divertissement et de la culture de masse, a été envisagée le plus souvent à travers la 
diversité des formes ludiques et des modèles récréatifs associés à la photographie foraine (Jézéquel 1995 ; Chéroux 2005). Quelques travaux ont élargi le spectre au-dehors des fêtes foraines, situées en milieu urbain, pour envisager le métier de ces artisans installés à l'occasion dans les foires des villages, les lieux de villégiature ou les sites du tourisme naissant de la fin $\mathrm{du}_{\mathrm{XIX}} \mathrm{e}^{\mathrm{e}}$ siècle, et qui circulaient de manière itinérante de la ville à la campagne, et inversement (Linkman 1990). Malgré ces études pionnières et isolées, la figure du photographe professionnel itinérant reste minorée et le sujet constitué par les pratiques de ce genre photographique demeure marginal en raison notamment de sa nature présumée instable. Un autre obstacle semble constitué par la difficulté de penser l'activité des opérateurs photographes en rapport avec une pratique personnelle de la circulation, une expérience de la mobilité et une condition qui voisine avec celle des migrants, qu'il s'agisse d'une itinérance au-delà des frontières nationales ou à l'intérieur d'un même pays.

2 L'intérêt modéré des historiens de la photographie pour cette profession repose sans doute sur une vision de la mobilité des photographes perçue nécessairement comme temporaire ou liée à l'exécution d'un travail qui les rattache à une base fixe, point de départ et de retour. Si toutes les grandes histoires de la photographie abordent la diffusion de la photographie par le biais des ateliers mobiles capables de se déplacer vers leurs sujets, l'idée d'une mobilité consubstantielle à l'activité du photographe reste abordée de manière très indirecte.

3 Le rappel de chapitres glorieux des premières décennies de la photographie insiste sur le caractère itinérant, par définition, de photographes à la fois explorateurs du globe et des grands événements de l'histoire (Lenman 2005 ; Maurice 2008) : le peintre Horace Vernet parcourt l'Égypte en 1839 et en rapporte ses célèbres Excursions Daguerriennes (Lerebours 1840-1843); un pionnier de la photographie en Suisse, Johann Baptiste Isenring, expérimente dès les années 1840 des procédés innovants et parcourt le Sud de l'Allemagne et la Suisse avec son studio mobile (Wäspe 1985); en 1855, Roger Fenton réalise les premières images d'une guerre, en Crimée, et transporte avec lui un studio sur roue dans son Photographic Van, dont l'image est diffusée immédiatement dans la presse, notamment dans le numéro resté célèbre de l'Illustrated London News (Anonyme 10 novembre 1855). Ces expériences inaugurent une pratique courante qui consiste à élaborer les moyens techniques d'un voyage destiné à la réalisation et au transport d'images photographiques rapportées, dans la plupart des cas, vers le siège des sociétés savantes, des journaux ou des institutions scientifiques des États qui financent, dès la fin des années 1830, ces expéditions photographiques.

La couverture photographique du réel s'effectue alors à partir d'une contrainte technique qui n'est pas sans implication: la fragilité du matériel et des supports photosensibles, la rareté des ressources transportées, l'organisation mesurée des gestes engagent l'esthétique des images produites et une mise en image du monde qui découlent de ces contraintes, comme cela a été étudié en particulier dans le cas du photographe voyageur Felice Beato (Osborne 2000; Caraion 2003; Lacoste 2010 ; Mullins 2012; Pinguet 2014). Surtout, ces expériences de photographies itinérantes constituent, à cette période, un capital de savoirs et de pratiques, relayé notamment par les revues spécialisées de photographie. Centré sur la fonctionnalité et la portabilité des instruments techniques, ce savoir cumulé se diffuse au même moment dans le domaine des usages commerciaux de la photographie, passage auquel 
contribuent de nombreux manuels comme celui de Léon Vidal consacré au «touriste photographe » (Vidal 1885-1889).

5 Ce déplacement semble perçu initialement comme une dégradation et la noblesse de l'art photographique paraît écornée par l'apparition des premiers photographes itinérants faisant commerce de leur pratique. En 1858, Ernest Lacan, grand promoteur de la photographie comme art, raconte dans un petit texte plein de colère l'apparition à Paris de ceux qu'il qualifie dédaigneusement de «saltimbanques de la photographie » (Lacan 1858). En dénonçant « les petits marchands qui vendent au rabais des épreuves de nature à dégoûter le public de la photographie», ce pamphlet rend compte des premières échoppes improvisées installées près du Châtelet, sur les espaces laissés en friche, ou au bois de Boulogne :

«À quelques pas de là, un homme était occupé à mettre en équilibre une sorte de guérite fermée, montée sur quatre roues, et dont la lucarne était garnie d'un rideau jaune. Nous le vîmes planter sur l'herbe quatre pieux sur lesquels il tendit une corde pour se faire une enceinte. Puis il plaça l'une des extrémités de cet atelier improvisé un écran pour lui servir de fond, posa devant une chaise sur laquelle il fit asseoir une femme qui l'accompagnait, et prenant son objectif, il mit au point, afin sans doute d'être prêt à fonctionner quand le public se présenterait. » (Lacan 1858)

Observateur involontaire de la naissance d'une pratique qui s'étend par la suite au monde entier, Lacan déplore le triste sort de ces photographes, dénonce leur ignorance, leur façon vulgaire d'attirer le client, leurs tarifs exorbitants et leurs manières d'escrocs. Mais il s'insurge principalement contre la piètre qualité esthétique des épreuves ainsi produites et expose, malgré lui, les raisons du succès à venir des photographes ambulants :

«Il est certain que l'on ne peut empêcher les saltimbanques de faire de la photographie, ni les photographes de se faire saltimbanques, ni le public de se laisser prendre à leurs annonces; mais il n'en est pas moins déplorable de voir un art que des hommes de talent ont porté si haut, traîné sur un champ de foire pour y disputer le succès aux enfants à deux têtes et [aux] avaleurs de sabres. » (Lacan 1858)

7 Promoteurs d'une telle monstruosité photographique, les ambulants sont ainsi décrits comme les intermédiaires d'une diffusion inexorable de la photographie à l'ensemble de la société, en dépit d'une qualité jugée discutable, voire condamnable, des images produites. L'inversion du dispositif de l'offre et le fait que les photographes se rendent désormais au-devant du public, plutôt que d'attendre derrière les vitres de leur boutique, constituent la particularité centrale de cette activité, décrite dans plusieurs cas comme un facteur essentiel dans la propagation des usages de la photographie, que ce soit dans le Far West américain, les Indes britanniques ou l'Afrique subsaharienne (Kailbourn \& Palmquist 2000; Spude 1989; Maurice 1995; Lee Eltzroth 2008; Mahadevan 2013 ; MacDougall 2005 ; Viditz-Ward 1987 ; Werner 1996).

Il sera question ici des métiers de la photographie ambulante exercés dans un cadre professionnel et commercial dont les ressorts, les procédés et les techniques, ne sont pas éloignés des pratiques des photographes voyageurs mais dont les usages diffèrent précisément parce que leur production se destine essentiellement au public des consommateurs et des acheteurs, qui échangent de l'argent contre une image, de soi ou des autres (Marcenaro 1995).

Comment définir cette activité professionnelle et les conditions quotidiennes de son exercice? Une documentation éparse, issue de la presse, des rares collections d'images 
et d'ouvrages spécialisés permettra de dresser un portrait du photographe ambulant dans les limites mouvantes d'une Europe des passages entre les années 1880 et 1930 : à la fois commerçant ambulant, entrepreneur d'une microéconomie de l'image, praticien caméléon et artisan habile et inventif, il est l'objet de descriptions ambivalentes qui contribuent à constituer le photographe ambulant en une figure importante des nouvelles pratiques populaires de la photographie.

\section{Devenir photographe ambulant : la naissance d'un marché}

Comme c'est le cas pour les photographes installés dans des studios, la transaction financière distingue cette activité car elle détermine un rapport strictement mercantile dans la production des images : les photographes ambulants vendent une image, plus ou moins désirée comme on le verra, et reçoivent une rémunération pour leur travail. Cet argent est alors réinvesti dans l'approvisionnement des outils d'une production future. À première vue, cette dimension économique ne semble pas distinguer les photographes ambulants de leurs collègues sédentaires, qui demeurent dans leur boutique dans l'attente du client, pourtant elles déterminent un équilibre original entre l'offre et la demande: l'ambulant n'apporte pas seulement les moyens d'une production, l'outillage technique et le savoir-faire, il transporte avec lui l'offre ellemême, il suscite par sa présence la possibilité d'une image et fait naître, voire impose, par la constitution d'un rituel, le désir d'une image de soi. En cela, le photographe ambulant n'est pas très éloigné du découpeur de papier noir qui dans une tente précaire composait, au XVII ${ }^{\mathrm{e}}$ siècle, les silhouettes des passants, ou bien de l'artiste qui se faisait dessinateur de rue et qui réalisait pour quelques sous un portrait rapide du tout-venant: Emma Rutherford évoque ainsi les silhouettistes itinérants qui œuvraient en Angleterre parfois alternativement dans les stations balnéaires, les villes moyennes

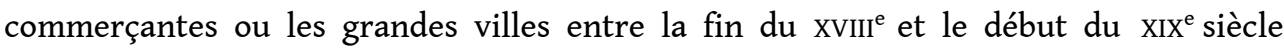
(Rutherford 2009: 155-171).

10 La nature de l'image photographique et la diffusion des images de soi et de leurs usages donnent à cette pratique une ampleur inédite qui touche toutes les couches de la société, dans toutes les grandes métropoles du monde et, peu à peu, dans l'ensemble des villages dans les campagnes et même, parfois, dans des lieux très reculés du monde rural. Le photographe ambulant suit d'ailleurs les chemins parcourus par les colporteurs et les commerçants ambulants des époques précédentes et profite des nouveaux modes de circulation pour s'inviter dans les réseaux changeants de la mobilité qui surgissent au XIXe siècle (Fontaine 1993, Rosental 1999). La diffusion des portraits photographiques en particulier et le succès de ces nouvelles images, stimulé par l'attrait de la bourgeoisie ascendante pour la mode du portrait photographique, portent naturellement le développement de la photographie ambulante mais ne suffisent pas à expliquer l'essor de cette profession, principalement à partir des années 1870 (Freund 2011 [1936], Gilardi 1976, Charpy, 2007).

11 L'attrait pour une profession à la fois spécialisée mais ne requérant qu'une rapide formation technique, laissant de longs temps libres et propices à la divagation, à la fois mobile et répondant aux aspirations au voyage, pouvait concerner des catégories particulières comme celles des artistes en début de carrière ou en quête d'inspiration ; elle pouvait aussi s'avérer lucrative pour des ouvriers spécialisés de l'imprimerie, par 
exemple, frappés par le chômage ou des jeunes hommes en rupture de ban - cette profession, sauf exception, apparaît comme essentiellement masculine (Werner 1997). Jules Girardin (1832-1888), auteur prolifique de romans pour la jeunesse dans les années 1870 et 1880 , entreprend de raconter les pérégrinations du "premier photographe ambulant que la France ait connu» et de son commis, un jeune homme sans famille, photographe occasionnel entraîné dans cette profession par un photographe de métier, M. Bouvat, décrit comme un artiste peintre sans talent dont la conversion est décrite en ces termes :

«M. Bouvat, forcé de reconnaître qu'il ne deviendrait jamais assez bon peintre pour vendre un de ses tableaux, résolut d'être artiste quand même et se jeta dans la photographie. Ayant remarqué que toutes les bonnes places étaient prises dans les villes, il eut une idée de génie, celle d'exploiter les campagnes. » (Girardin 1909 [1889]: 52)

13 Figure centrale de ce roman de formation, Bouvat est présenté comme un artiste distingué et présentable, un photographe expert et un commerçant florissant et apprécié. Abandonné par ses commis précédents, il rencontre le jeune Philippe et lui présente les avantages de sa condition :

«Je ne suis pas photographe à demeure, installé dans une ville, sauf pendant la mauvaise saison. Tant que durent les beaux jours, je suis photographe ambulant. Je vais de ville en ville, de village en village, de foire en foire. Comme je fais toujours la même tournée, on me connaît partout, et je puis bien dire que tout le monde m'estime ; le métier est bon, puisque tel que vous me voyez, j'ai chez mon banquier quatre-vingt mille francs qui ne doivent rien à personne. » (Girardin 1909 [1889] : 59)

14 Pour rassurer encore davantage son futur assistant, il prend bien soin de distinguer sa condition de celle des autres forains et donne des gages de sa réputation :

«Nous ne sommes point des saltimbanques et nous ne fréquentons pas les saltimbanques (...). J'ai une grande voiture à moi qui ne ressemble pas aux caravanes des faiseurs de tours, je vous en réponds. C'est là-dedans que j'opère quand le temps menace, et sous une tente quand il fait beau. Depuis vingt ans que je tourne dans le même cercle, ma place est assurée d'avance partout, et toujours dans un endroit éloigné de la grosse foule. » (Girardin 1909 [1889] : 59)

Ces arguments composent la matière du roman qui raconte les mésaventures $d u$ photographe et de son commis sur les routes de France, dans la roulotte conduite par un cheval appelé malicieusement Collodion. Probablement informé par un homme du métier, Girardin évoque dans le détail l'organisation idéale de la roulotte d'un photographe, sa connaissance cumulée des espaces et des coutumes, son ingéniosité déployée pour attirer l'attention des fermiers réticents, des notables en vacances, des soldats de passages et des premiers touristes balnéaires sur les plages du bord de mer. Le talent du commis et l'expérience du photographe conduisent au succès de leur entreprise et à l'installation d'un "atelier d'hiver» qui attire aussitôt la clientèle. L'histoire raconte l'émancipation du commis qui apprend le dessin et devient, par un étrange retournement de situation, un peintre célèbre, le métier de photographe ambulant étant ainsi caractérisé comme une activité incertaine et aléatoire mais aussi portée par les promesses du succès (fig. 2). 


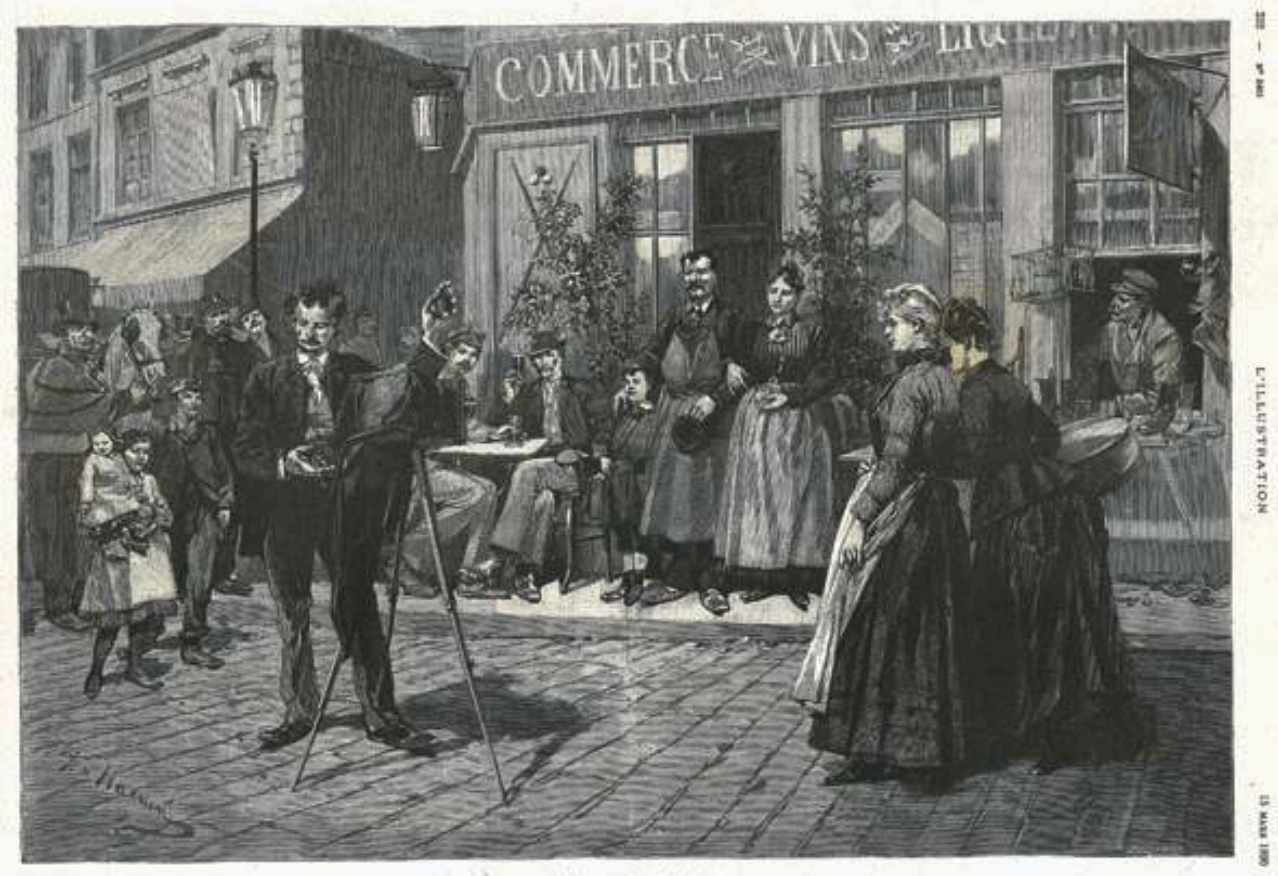

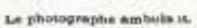

Gravure parue dans L'Illustration, $n^{\circ}$ 2455, 15 mars 1890, p. 232, Collection particulière.

Frédéric de Haenen (dessinateur), Albert Bellenger (graveur)

\section{Les conditions d'une mobilité spatiale et professionnelle}

Le photographe ambulant se définit comme une personne voyageant avec un appareil photographique et des instruments de prise de vue ainsi que des supports photosensibles, puis à partir des années 1870, des ustensiles de développement, dans le but d'exercer son métier de photographe et avec l'intention de vendre sa production photographique à des consommateurs, ou parfois de l'échanger. Suivant cette définition, la route exerce un attrait spécifique comme source de profit: le déplacement d'un village ou d'un gros bourg à un autre, dans le monde rural, ou d'un quartier ou d'une rue à une autre, en milieu urbain, garantit le renouvellement $\mathrm{du}$ marché ciblé (fig. 3). 


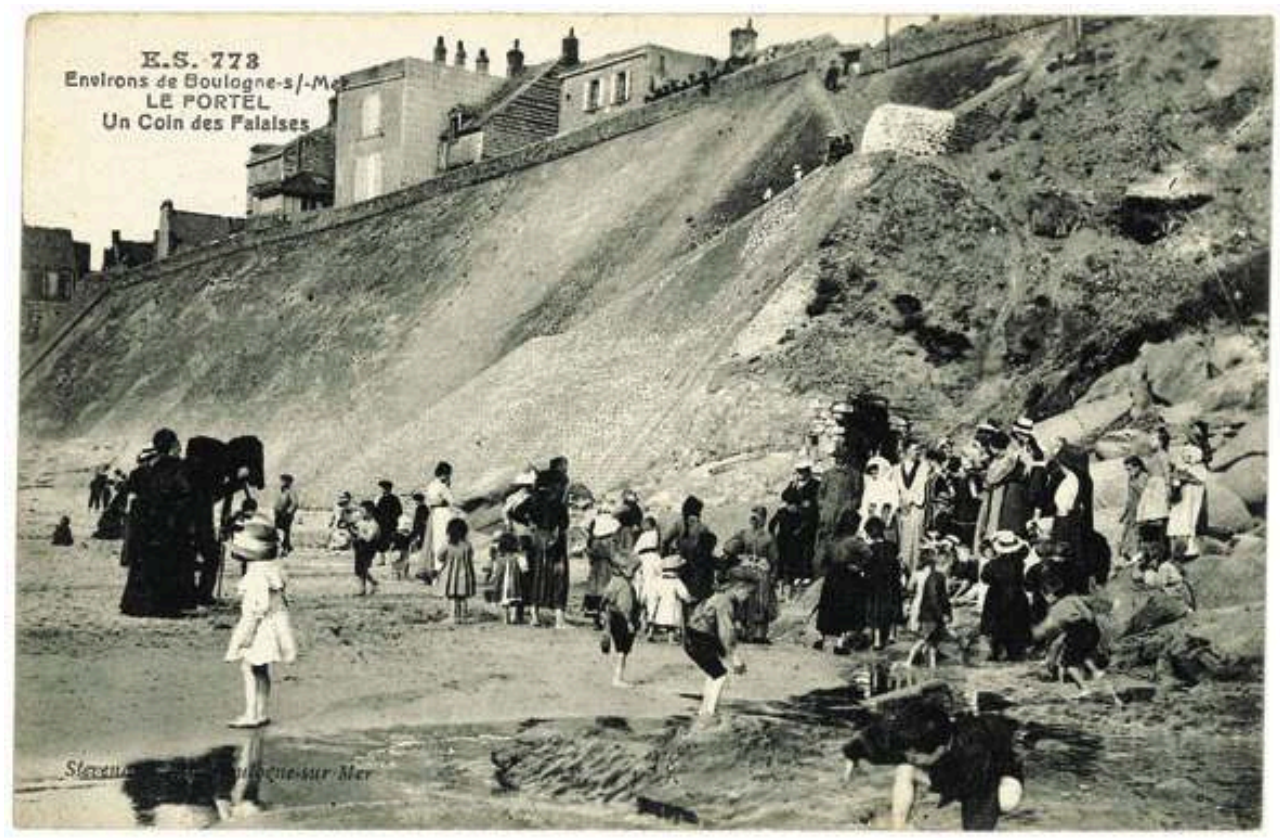

Un coin des falaises [Groupe posant devant un photographe ambulant], Carte postale, 9x13, ca. 1910, Collection particulière.

Stevenain (éditeur)

Mais cette activité pouvait aussi être pratiquée en alternance avec la conduite d'un studio sédentaire durant des périodes d'itinérance ou, de manière occasionnelle, lors d'événements particuliers ou suivant les saisons, obligeant le photographe à quitter son studio temporairement et à utiliser ce studio comme atelier de travail lors de ses retours. Le cas de François Gabriel est à ce titre exemplaire: ancien courtier en assurances, celui-ci ouvre son atelier boutique dans les années 1920 dans une boutique située aux bords des escaliers de la rue Muller à Montmartre ; parfois il s'installe aux pieds des marches, lorsque le temps le permet, avec un appareil portatif (Lacroix 2011). Photographe de studio, de quartier et ambulant en alternance, il travaille pendant plusieurs décennies, jusqu'aux années 1950, au service des habitants du quartier et des passants, son travail constituant aujourd'hui une sorte de mémorial photographique des escaliers de Montmartre. De multiples configurations pouvaient donc exister mais la possession d'une boutique pour un photographe ambulant traduisait une aisance et une stabilité d'emploi que ne possédaient pas les autres opérateurs, mobiles en toutes circonstances, s'approvisionnant, en ville ou par correspondance, lorsque leurs stocks étaient épuisés (Hucq-Lajolie 1983).

Pour montrer la difficulté d'une définition stricte et générale, il suffit de rappeler le récit du grand helléniste Victor Bérard, dans son ouvrage sur Les Phéniciens et l'Odyssée publié en 1902. Pour évoquer les "roulottiers de la mer primitive» celui-ci fait référence au commerce ambulant de la France rurale, qui oscille selon ses termes, du temporaire au permanent :

«(...) Les porte-balles et marchands forains arrivent avec leur camelote sur leur dos ou dans leur roulotte; ils ouvrent [une] boutique provisoire et finissent par demeurer des mois et des années. J'ai vu dans mon enfance, vers 1872, arriver à Morez-du-Jura un photographe ambulant qui installa près de sa roulotte (...) un 
grand atelier en plein-vent et une boutique : trente ans après, il est toujours là,

campé dans sa voiture qui lui sert de maison. » (Bérard 1902 : 399)

d'activité des opérateurs. Ils portent avec eux un capital de savoirs et de techniques et conquièrent des territoires en suscitant une demande et en apportant une offre en images et en pratiques visuelles parfois rares ou inconnues. Ils sillonnent les mêmes territoires, constituent une clientèle dans un espace connu et maîtrisé et dans un temps parfois long qui permet de fidéliser les consommateurs. Dans le monde rural, le passage d'un de ces photographes marque un événement et le souvenir de cette étape reste pour longtemps à la fois dans les souvenirs et, par les traces visuelles laissées derrière lui, dans les tiroirs des commodes, les portefeuilles, les albums et les petites boîtes où s'entassent les rares images de soi qui sont conservées avec soin (Maresca 2004). Des témoignages demeurent de ce rôle intermédiaire des photographes ambulants, véritables incarnations du transfert culturel (Jowanowitch 1903: 278; Steen 1987; Hartley 1994).

L'autre paramètre essentiel d'une définition de cette profession tient dans la place de cette activité dans le parcours professionnel des photographes. Elle pouvait s'inscrire dans une combinaison variable de pratiques professionnelles : le travail spécialisé du photographe de métier, la pratique occasionnelle combinée à une activité stable différente, la pratique de la photographie parmi d'autres pratiques professionnelles dans un système de pluriactivité, enfin la pratique temporaire, après ou avant l'exercice d'autres professions. La littérature sur le sujet évoque toute sorte de professions complémentaires: ingénieur, dessinateur, hypnotiseur, vendeur d'instruments de musique, assureur, chasseur et même prêtre. À l'extrémité de cette chaîne de possibles, l'ancrage familial de la photographie ambulante s'établissait dans de véritables dynasties de photographes. Le caractère très technique et spécialisé de cette profession, sa dimension commerciale, située dans un espace parcouru régulièrement et fondée sur une clientèle dont les attentes s'échelonnaient tout au long de la vie, favorisaient en effet une transmission dynastique de ce métier. Le cas le plus emblématique est sans doute celui du photographe allemand Trudpert Schneider (1804-1899), ancien peintre miniaturiste, qui apprend la technique du daguerréotype auprès d'un photographe itinérant français, Joseph Broglie, et prend la route avec ses fils, Heinrich (1835-1900) et Wilhelm (1839-1921). Ils se spécialisent en particulier dans la production de vues stéréoscopiques enchâssées dans de luxueux écrins qui se destinent à une clientèle fortunée. Entre 1847 et 1865, les photographes Schneider parcourent ainsi l'Europe des capitales germaniques vers l'Autriche, l'Italie du Nord et même la Russie ; à la même époque, sur un modèle similaire, les frères Renard circulent entre le Danemark et l'Allemagne (Morand 2001 ; Steen 1993).

\section{Portraits de photographes ambulants}

Un grand nombre de photographes ambulants partageait leur activité avec le travail d'artiste rémunéré, dessinateurs le plus souvent, graveurs ou peintres. Le parcours d'un caricaturiste français renommé de la Troisième République, comme le boulangiste, puis 
antidreyfusard, Alfred Le Petit (1841-1909), ne semble pas isolé. Fils d'un horloger, il quitte sa famille pour se consacrer au dessin et exerce une foule de métiers parmi lesquels, dans les années 1860, celui de photographe ambulant. Durant ses tournées, il pratique le dessin et apprend à regarder les scènes de la vie quotidienne avant de se consacrer à la peinture puis d'être engagé par des journaux comme Le Grelot ou Le Charivari (Normandy 1931 ; Potvin 1987 ; Aa. Vv. 2005). D'autres parcours démontrent que cet emploi était souvent exercé par des personnalités hors du commun, aux marges de la norme sociale, des atypiques qui étaient à la fois débrouillards, inventifs mais aussi, un peu ou beaucoup, roublard et rusé, comme ce photographe d'Indre, fantasque et solitaire, surnommé "Le Toqué » (Chatain 1992). Des témoignages épars indiquent que les photographes ambulants pouvaient aussi appartenir aux communautés roms (tsiganes) comme l'a relevé Frank Cuttriss, lui-même photographe, dans son ouvrage Romany Life consacré aux familles rencontrées au Royaume Uni avant la Première Guerre mondiale (Cuttriss 1915). Des contacts avec des Sinti italiens sont aussi avérés dans la vie et l'œuvre de plusieurs photographes ambulants du Centre nord de l'Italie (Censi 1987 : 37, 66-69 ; Borhi \& Fabbri 1991).

Comment mieux traduire cet aspect de la condition des photographes ambulants sinon en citant plusieurs expériences comme celles de Nicolas Wickelson ou Panaït Istrati ? Le premier est né en 1892 à Tallin, en Estonie, et est issu d'une famille de la bourgeoisie russe, disloquée au moment de la révolution de 1917. Alors jeune officier, il est contraint à l'exil et traverse le monde, de la Chine à l'Argentine, avant de s'installer en Corse. Il exerce alors de nombreux métiers, mécanicien, jardinier, électricien, plombier, ouvrier agricole, et se trouve finalement employé à l'Hôtel de France d'Ajaccio. À l'âge de 45 ans, il adopte le métier de photographe ambulant et s'installe à son compte en 1937. En compagnie de sa femme qui l'assiste dans son travail, il exerce pendant plusieurs décennies dans la région d'Ajaccio et se présente aux clients par cette formule extravagante : « Faites-vous photographier par Nicolas, officier du Tsar » (Fieschi 2002).

Panaït Istrati (1884-1935), écrivain d'origine roumaine, découvert par Romain Rolland, vécut l'errance à travers la Méditerranée aux temps de la Première Guerre mondiale. Tour à tour apprenti chez un cabaretier puis chez un pâtissier en Roumanie, marchant ambulant, puis soutier dans des paquebots, il échoue à Nice dans l'après-guerre et $\mathrm{y}$ devient photographe ambulant. Lors de la sortie de son livre Kyra Kyralina en 1924, un entretien paraît dans la presse où il évoque son travail, aux limites de la légalité :

«Sur la promenade des Anglais, ses pareils sont bien une douzaine, qui, secondés par vingt marchands de tapis, sollicitent et harcèlent le client, en dépit de toutes les interdictions municipales. De temps en temps, la police intervient. Elle emmène au poste l'un ou l'autre des commerçants trop zélés, que l'on relâche d'ailleurs bientôt. Les premières fois, la contravention leur coûte une amende; ensuite ce sont des jours de prison.

- J'en ai fait douze, avoue Istrati souriant. Mais j'ai eu quelques beaux clichés : le shah de Perse, le roi de Siam, et, en avril dernier, S. M. la reine de Roumanie. À ce moment, j'ai rencontré à Nice le directeur de l'Universul, le grand journal de Bucarest, et je suis devenu son correspondant à Paris. Le métier de photographe mène à tout... Mais je retournerai bientôt à Nice, voir les "copains"... " (Anonyme 12 juin 1924)

De l'obscurité des prisons à la lumière des têtes couronnées, des rédactions de grands journaux au monde de la littérature, Istrati incarne cette figure ambivalente du photographe ambulant, à la fois aventurier et artisan d'images. Le panneau publicitaire, en plusieurs langues, qu'il utilisait pour marquer son territoire sur la Promenade, 
indiquait "Photo plein air - Silhouettes - Les photos sont livrables le lendemain de la pause". Cette image conservée dans les archives témoigne, avec quelques fragments écrits, d'une activité incessante et épuisante passée à proposer ses services (fig. 4) :

«Je rentre dans ma chambre après avoir patrouillé des heures sur la Promenade, traqué par des agents, embêtés par les clients qui ne sont pas des clients, et surtout assommé par mon cafard. Je n'en peux plus ! - «Messieurs, Mesdames, des belles photos, $8 \mathrm{~F}$ les six...» - Oh, que c'est bête ! Oh, que c'est ignoble!» (Brezu Stoian $1984: 41)$

Portrait de Panaït alors qu'il était photographe ambulant à Nice en 1923

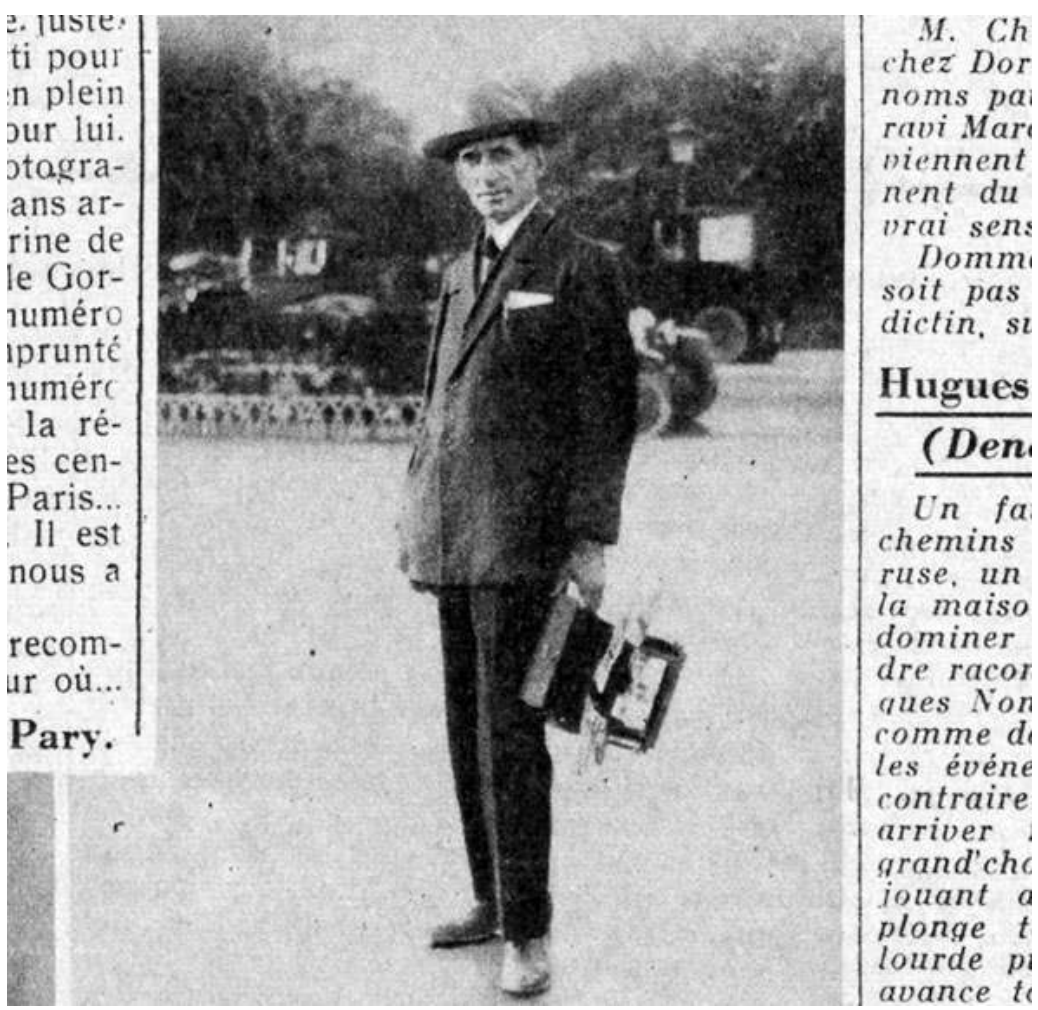

Photographie parue dans Marianne, $n^{\circ} 131,24$ avril 1935, p. 4, Bibliothèque nationale de France, JOD-127.

Auteur inconnu

La répétition incessante des gestes et des sollicitations, le travail en plein air, le caractère incertain des revenus quotidiens et la précarité économique concourent à l'épuisement physique et conduisent au découragement. Ce témoignage évoque aussi la position légale du photographe ambulant qui était soumis, en France, au port de la patente suivant la loi du 15 juillet 1880 sur les professions commerciales. Le coût induit par ce certificat et les obligations fiscales qui en découlaient favorisèrent les pratiques illégales et de nombreux photographes ambulants qui n'étaient ni employés, ni propriétaires d'un studio tentaient d'échapper à cet impôt (Bigeon 1893 : 137). Dans les années 1900, le renforcement des mesures de contrôle à l'encontre des professions ambulantes ne peut empêcher la multiplication des opérateurs illégaux qui répondent à l'amplification de la demande. Les réclamations des photographes installés envers les photographes ambulants en infraction et cette concurrence déloyale suscitent d'ailleurs, au début des années 1910, des tensions de plus en plus palpables et les autorités publiques se trouvent interpellées par les photographes professionnels 
regroupés en syndicats (Anonyme 1913). Pour les resquilleurs, la capacité d'échapper au contrôle des policiers et de passer entre les mailles de la surveillance venait compléter la panoplie des qualités requises pour devenir photographe ambulant.

\section{Le métier au quotidien}

La dimension quotidienne du travail de ces opérateurs se traduisait par des conditions d'exercice et des pratiques spécifiques qui formaient un habitus professionnel à part. Ils se déplaçaient le plus souvent accompagnés d'un chariot à bras, mais aussi parfois à pieds, en bicyclette, et le plus souvent avec un wagon mobile attelé par des chevaux ou des bœufs; des témoignages signalent des photographes ambulants circulant dans des wagons ferroviaires privatisés aux États-Unis. Mais la plupart portent sur l'épaule, d'un lieu à l'autre, les quelques outils de leur studio (fig. 5).

\section{Deauville 1921}

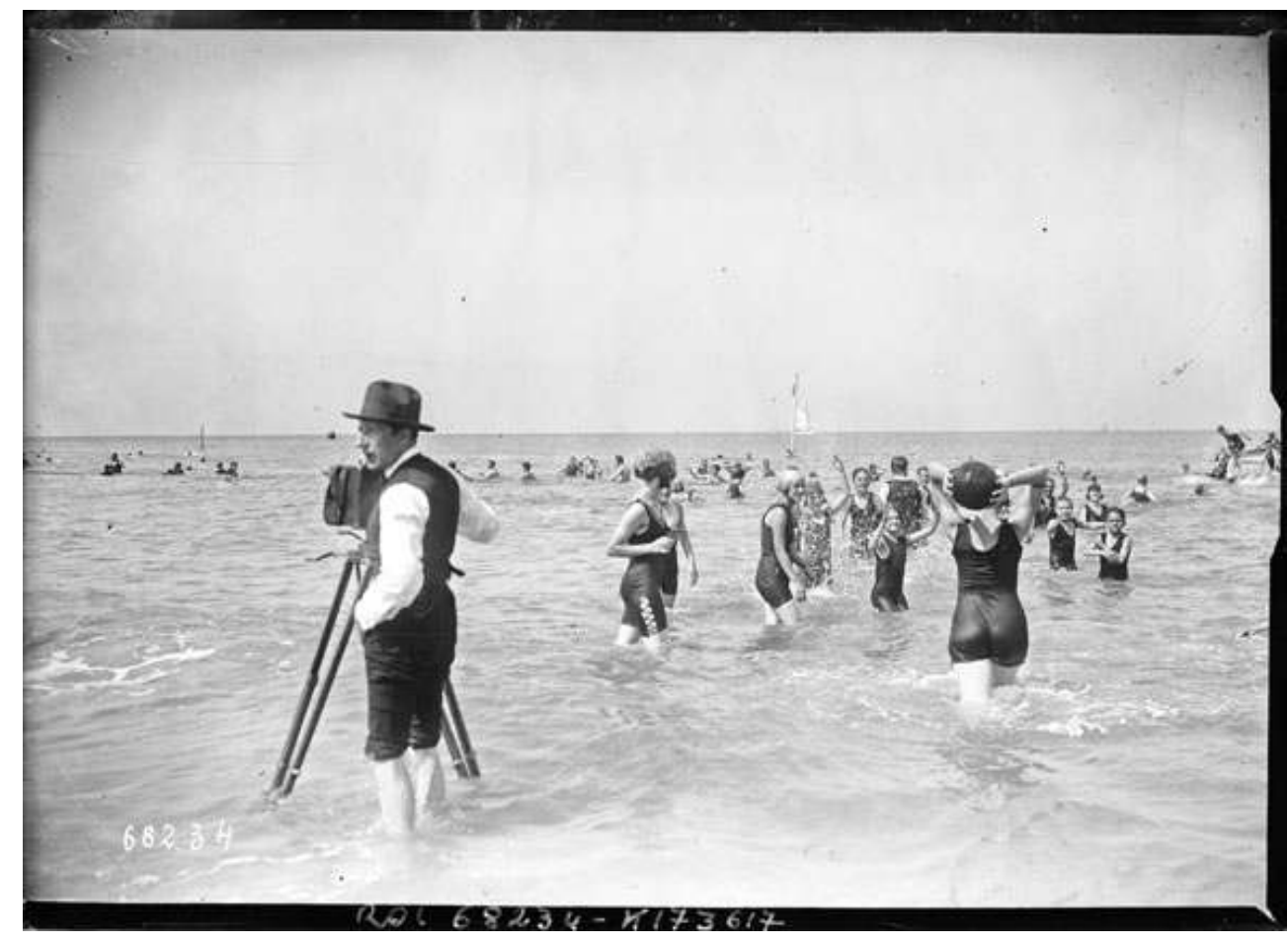

[Un photographe ambulant parmi les baigneurs], Négatif sur verre, 13x18, 1921, Bibliothèque nationale de France, El-13(816).

Auteur inconnu (Agence Rol)

Ils disposent souvent d'une tente pliante, d'une mallette de transport et préparent leur arrivée en envoyant à l'avance des annonces dans les journaux locaux publiés dans les régions et les villes de passage. Certaines roulottes, qui servent de logement, sont entièrement équipées d'un studio couvert de prise de vue mais dans d'autres cas, il peut s'agir d'une simple charrette recouverte de toile (Aa. Vv. 2013 : 38-41). Le plus souvent, le photographe installe un espace étroit, démontable et provisoire, réduit à l'état d'un simple tissu accroché à un mur, d'un faux plancher, d'un guéridon, la seule profondeur de champ et les fils invisibles partant de l'objectif constituant à eux seuls les limites de ce studio provisoire. 
Plusieurs témoignages mettent l'accent sur les systèmes élaborés pour mener à bien cette activité et évoquent l'attirail du photographe, sa recherche de la clientèle, les conditions de prise de vue. Un article de presse de 1904, consacré aux métiers parisiens ignorés, évoque avec précision la journée de cet « industriel » qui procède, dit-on, avec la collaboration du soleil :

«Il déambule des hauteurs dès le matin, traînant sa voiturette fermée, qui contient son matériel artistique. Deux ou trois employés, l'album sous le bras, l'escortent, ce sont des courtiers rabatteurs qui, tout à l'heure, iront de rue en rue, de maison en maison et de porte en porte, faire l'article, pour engager les indécis à livrer leurs têtes au miroir de la plaque sensible, au prix d'un franc cinquante, cadre doré compris.

Pendant que ses commis sont à la chasse aux têtes, l'opérateur a déballé ses instruments, et bientôt le chevalet se dresse, intriguant le petit peuple turbulent. En attendant le client sérieux, le patron choisit parmi les bambins un groupe qu'il dispose et qu'il fera à l'œil - c'est la carte d'échantillons qui servira de miroir aux alouettes pour les autres mamans. » (Legentil 1904) placée au service du photographe et les artifices utilisés pour créer l'événement (Nadernhy 1893) (fig. 6).

\section{Paris pittoresque. Le photographe ambulant}

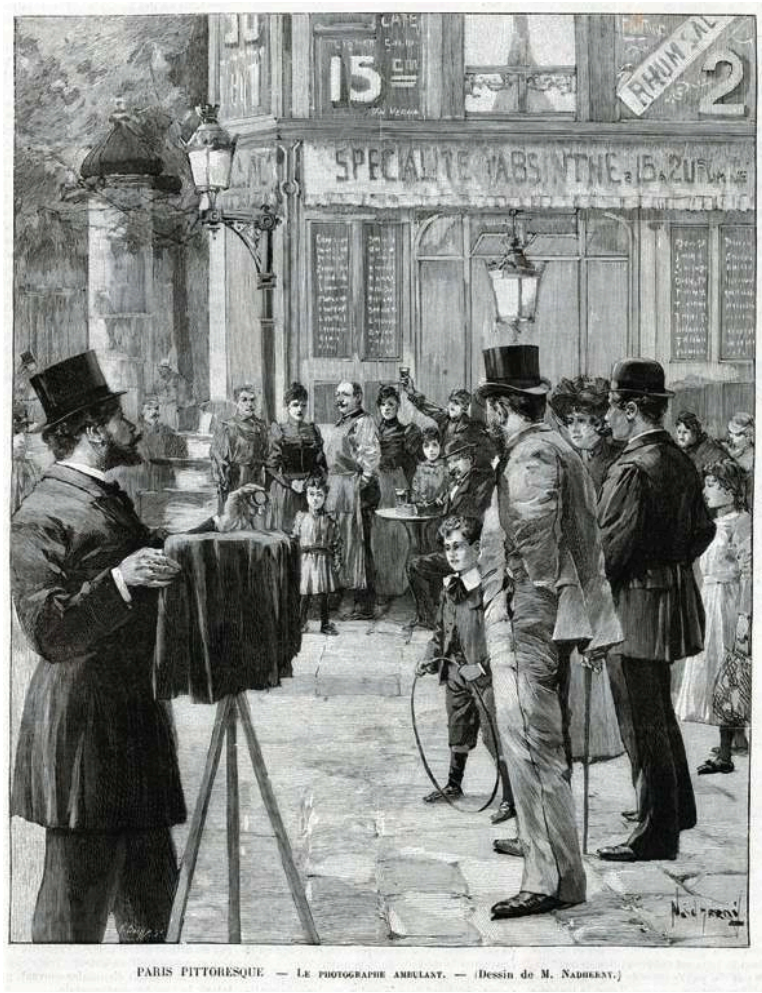

Gravure parue dans Le Monde illustré, $n^{\circ}$ 1890, 17 juin 1893, p. 381, Bibliothèque nationale de France, FOL-LC2-2943.

M. Nadernhy (dessinateur), H. Dochy (graveur)

Il est ensuite question du patron de la charcuterie qui fait poser tout son personnel devant la boutique, l'école maternelle qui profite de l'occasion pour réaliser une 
photographie de classe, les communions qui forment le fonds de commerce inépuisable de ces entreprises, les portraits à la naissance ou après décès.

Un autre témoignage de ces stratégies et de leurs effets est offert par l'auteur d'une courte enquête à valeur ethnographique sur les petits métiers dans la ville de Cognac. Il évoque lui aussi le métier des photographes ambulants qui par leur simple présence parviennent à susciter un désir d'image (Martin-Civat 1975 : 33-34). Le passage des opérateurs provoque soudainement une envie qui fractionne le temps, interrompt le temps du travail ou vient donner un sursaut au temps libre. Le contraste entre la simplicité du geste et la valeur affective intense des souvenirs photographiés semble par ailleurs rehausser encore l'importance de ce temps suspendu.

\section{Une figure sociale ambivalente}

À travers ces différents témoignages, le photographe ambulant se présente comme un négociant aguerri, qui invente des formes commerciales inédites jouant avec le désir d'images de soi, et qui parvient à susciter ce désir par toute une série de ruses. Ce sont ces qualités qui justifient des jugements grinçants à propos de cette profession considérée pour ses vertus d'amuseur et aussi ses défauts, sa roublardise et ses exagérations : une veine comique cible même cette profession particulière, comme en témoignent les élucubrations, à vrai dire indéchiffrables d'un humoriste sous le Second Empire (Löwe 1866). Parfois même, la figure de cet ambulant est choisie pour illustrer le fléau présumé du "vagabondage" tel que la littérature réformatrice de la fin du $\mathrm{XIX}^{\mathrm{e}}$ siècle le décrit en évoquant les périls qui menaceraient la sérénité dans les campagnes (Wagniart 1999). Le photographe ambulant se trouve décrit suivant une série de stéréotypes qui insistent sur son état physique et sa condition, sa pratique et son usage quotidien de produits chimiques, de liquides aux couleurs improbables, de papiers et de supports humides. L'auteur d'un feuilleton décrit en 1868 l'un de ses personnages en employant une formule symptomatique de cette image sociale relativement négative: "C'était un homme barbu et sale comme un photographe ambulant » (Calilas $1868: 3$ ).

Plusieurs caricatures parues dans la presse illustrée, en France, traduisent également l'ambivalence de cette figure sociale (fig. 7). 


\section{GROQUIS RUSTIQUES, - par PAUL LÉONNEC.}

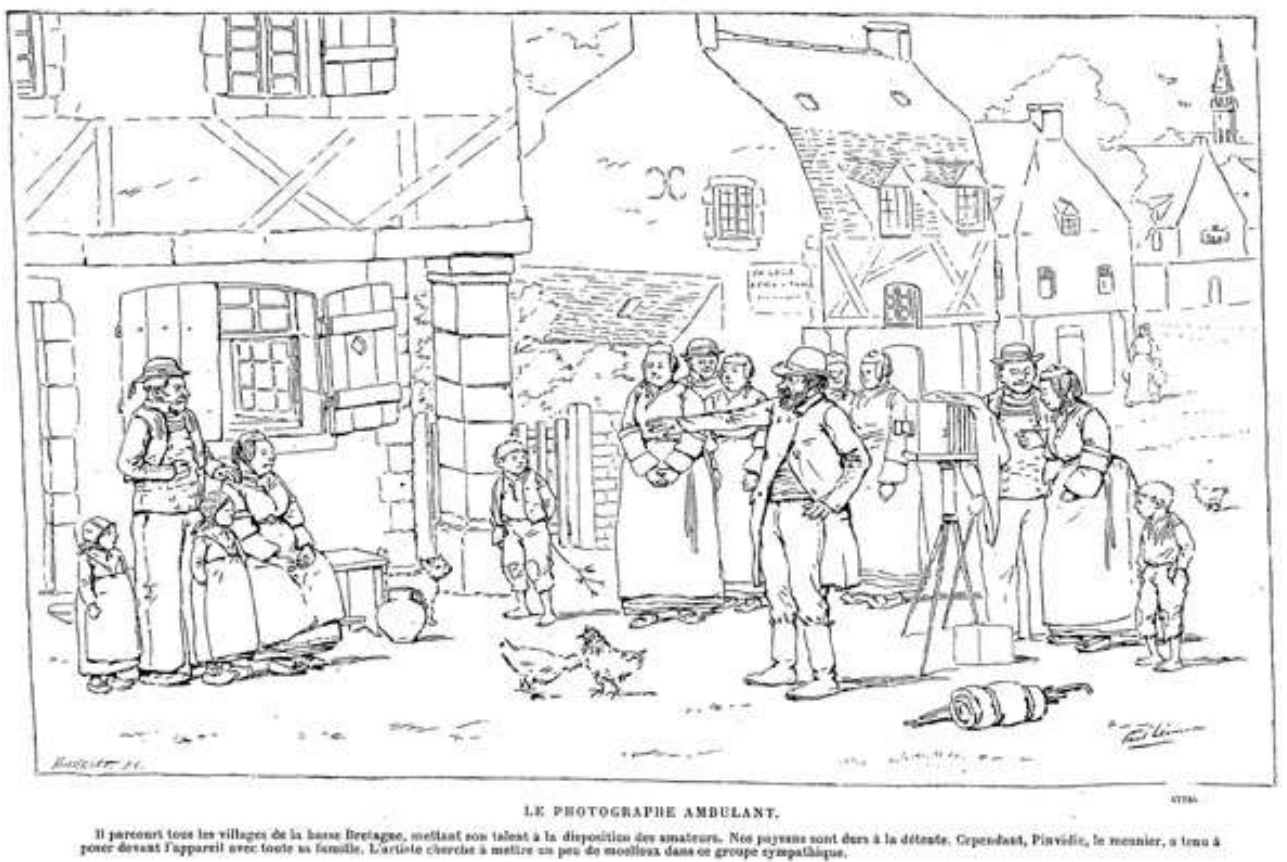

Gravure parue dans Le Journal amusant, n 1871, 9 juillet 1892, p. 2, Bibliothèque nationale de France, LC2-1681.

Paul Léonnec (dessinateur), Michelet (graveur)

31 En 1883, le dessinateur Trock montre, dans le journal parisien et populaire, La Caricature, le passage du photographe ambulant dans un petit village perdu, dessin légendé en ces termes :

«La concurrence lui a rendu impossible le séjour des grandes villes, où le photographe qui se respecte doit avoir des salons et des larbins dorés sur tranche, et couvrir des murs de réclames flamboyantes (...).

Il opère enfin par tous les temps, et tous les temps "sont préférables". Du reste, il n'est pas trop à plaindre pour l'instant. Le voici à Fouilly-les-Asperges. M. le maire a commencé par lui commander le portrait de sa vieille maison dont il a le bon goût d'être fier, n'ayant pas de quoi s'en offrir une neuve. Sensation sur la place publique. Les naturels n'ont rien de plus pressé que d'obstruer la vue, avec cet intelligent amour des arts que professent les masses à Fouilly-les-Asperges. D'aucuns cependant voient avec regret cette invasion du modernisme dans l'honnête cité d'ordinaire si paisible. » (Trock 1883)

Le photographe satisfait ici la vanité des petits notables de province, amuse les villageois présentés comme ignorants, usurpe le statut d'artiste et exerce une profession sur laquelle pèse le soupçon permanent de l'escroquerie, tout en incarnant, un peu malgré lui, une forme de modernité. L'image insiste par ailleurs sur la forme surprenante et ridicule du photographe montrant son derrière, caché sous son drap noir et dont la tête est remplacée par un objectif.

Dans son étude sur les photographes d'identité à Tananarive, dans les années 1980, Christian Papinot a souligné «cette intelligence des petits moyens » mobilisés par ces artisans (Papinot 1987 : 30 ; Papinot 1996 ; Nimis 2005 : 114-120). Il a montré comment cette profession a réussi à se maintenir grâce à la préservation des secrets professionnels et la transmission de «trucs» du métier, révélant ainsi le sens 
particulier de l'acte créatif attaché à la profession des photographes ambulants. En suivant les pas de Michel de Certeau, étudier l'invention du quotidien des photographes ambulants peut permettre de croiser la création graphique des objets photographiques et la dimension pratique d'une existence fortement marquée par sa dimension matérielle (Certeau 1980). En considérant l'activité professionnelle des métiers itinérants comme une expression de la créativité des gens ordinaires, il est possible en effet de rendre compte de cette liberté buissonnière des pratiques et de sa présence dans l'exercice quotidien de métiers qui exigent une adaptation constante aux lieux, aux objets ou aux climats. Le recours à l'idée d'une ruse créatrice s'impose aussi pour expliquer l'invention au quotidien de techniques visuelles adaptées aux contraintes, placées dans une zone de tension qui hésite entre la recherche de moyens de subsistance et le raffinement du geste, la perfection des mouvements et le souci du détail. Le recours à des stratégies originales mais incertaines pour séduire le regard et attirer l'attention résulte d'un savoir composite assemblé par des photographes qui cumulent toujours d'autres activités et d'autres expériences, qui les font ressembler aux saltimbanques et amuseurs de rue tour à tour jongleur, chanteur et avaleur de couteaux. En 1920, un entrefilet du Figaro tresse les louanges d'un de ces expérimentateurs, à la fois artiste et artisan, dans ce cas un poète et photographe ambulant :

«Il s'appelle Joseph (...) et s'intitule le Papillon nomade. Tout simplement! Il va de région en région et édite en cartes postales les sites qui lui ont plu. La vente de ces cartes postales suffit à le faire vivre. Sous chaque photo, il imprime des vers, de lui. Des vers didactiques ou solennels. Et c'est assez original. » (Anonyme 31 octobre 1920)

Cette mention parait significative de la position particulière de la production des photographes ambulants : entre production commerciale et production artistique, ces photographies résultent d'une élaboration systématique, et qui répondent en même temps à une volonté du public d'obtenir une image conventionnelle.

L'analyse des formes visuelles, d'une très grande diversité, prises par la production des photographes ambulants nécessiterait une étude à part. Quelques éléments peuvent être évoqués cependant pour souligner l'originalité des productions issues de leur travail. Pour différencier sa production et rompre le rythme répétitif d'une activité marquée par son caractère sériel, le photographe utilisait le temps parfois long qui suspendait son travail par nécessité - la pluie, le vent, la neige, la nuit - pour inventer des formes visuelles innovantes. Lorsque le photographe n'exerçait pas une autre activité, il pouvait en effet recourir à des microtechniques, si l'on peut dire, employées à toutes les étapes de la production, jusqu'à l'écriture de poèmes au dos des images : un angle de prise de vue, l'utilisation d'accessoires insolites, l'indication au sujet des gestes à accomplir devant l'objectif et relevant plus généralement de la position des corps, des signes apposés sur le tirage, l'usage de caches, de cadres, de légendes, de marques, d'inscriptions de toutes sortes. Le détour des images, par exemple, relève parfois d'une poétique du détail qui s'exprime dans ces caches multiformes découpés au couteau pour embellir les portraits et qui orne parfois sans raison les portraits des passants.

Il serait ainsi trompeur de considérer cette pratique d'images comme une forme élaborée ou aboutie de création et il paraît important de souligner que cette production se distingue par un caractère de très grande précarité, propice aux miracles du quotidien mais aussi, plus souvent, à une infinité de ratages : surexposition, bougé, flou, décadrage, recouvrement, incongruités en tous genres, déchirures, pliures, taches de 
noir, de blanc, de couleur, salissures multiples. La liste des ratages et des imperfections possibles est infinie et il serait d'ailleurs intéressant d'observer comment le public s'approprie certains de ses supposés défauts et transforme ces erreurs photographiques en objet du quotidien (Chéroux 2003 \& 2013).

Passeurs d'images et intermédiaires technologiques, les photographes ambulants garantissent la diffusion des pratiques photographiques à de nouveaux segments de la société. Mais ils favorisent aussi l'établissement de la photographie comme pratique collective, ludique ou familiale et contribuent à instaurer un habitus photographique et le partage à grande échelle de cette gestuelle de l'image photographique, dans la prise d'image ou la pose, qui s'impose aujourd'hui comme une évidence. Quel que soit le résultat produit, imparfait selon les standards de l'art photographique, les tirages élaborés composent les objets d'une photographie populaire qui s'établit peu à peu, dès la fin du $\mathrm{XIX}^{\mathrm{e}}$ siècle, comme un élément constitutif de toute sociabilité, privée ou publique.

\section{BIBLIOGRAPHIE}

Aa., Vv. 2013 L'Atelier d'un photographe de la fin du XIX siècle en maquette. Vevey : Musée suisse de l'appareil photographique.

- 2005. Alfred Le Petit, photographe "maudit". Négatifs commentés. Paris : Éditions Céros.

Anonyme. 10 novembre 1855 « M. Fenton's Crimean Photographs », Illustrated London News 769 : 557.

Anonyme. 1913 « Les Photographes ambulants et la patente ", L'Information photographique. 12 : 265.

Anonyme. 31 octobre 1920 « Papillon, dit le Nomade », Le Figaro.

Anonyme. 12 juin 1924 « L'Étrange figure de Panaït Istrati, conteur oriental de langue française », Le Petit parisien.

Armand, P. 2011 « La Carte photo privée... de studio et de l'amateur », Cartes postales et collections $249: 3-16$.

Baudrain, F. 2013 Photographie foraine et ferrotypie, quand un genre photographique s'approprie un procédé. Étude expérimentale des vernis en termes de formulation et de protocole d'application, Master. Paris : École nationale supérieure Louis Lumière.

Bérard, V. 1902 Les Phéniciens et l'Odyssée, vol. 1. Paris : Armand Colin.

Bigeon, A. 1893 La Photographie et le droit. Paris : Charles Mendel.

Borghi, G. P. \& Fabbri L. 1991 « Giravano con le carovane con i cavalli. Immagini di un fotografo ambulante della campagna bolognese », Lacio Drom 27(1) : 27-33.

Brezu Stoian, C. dir. 1984 Panaït Istrati. Fotograf şi în fotografii / Photographe-photographié, trad. I. Herdan. Bucarest : Meridiane. 
Calilas, H. de 1868 « Notre grand monde », L'Éclair 2 (20) : 2-3.

Caraion, M. 2003 Pour fixer la trace. Photographie, littérature et voyage au milieu du XIX siècle. Genève : Droz.

Censi, M. 1987 1910-1920 a Cento. Dalle fotografie di Gaetano Falzoni-Gallerani. Cento : Siaca arti grafiche Giovanni Tassinari.

Certeau, M. de 1980 L'Invention du quotidien, 1, Arts de faire. Paris : Union générale d'éditions.

Charbonnier, J.-P. \& Soupault P. 1957 Chemins de la vie. Monte-Carlo : Éditions du Cap.

Charpy, M. 2007 « La Bourgeoisie en portrait. Albums familiaux de photographies des années 1860-1914 », Revue d'histoire du XIX ${ }^{e}$ siècle 34 (1) : 147-163.

Chatain, G. 1992 Antoine Coudert, 1866-1910. Photographe ambulant. St-Benoit-du-Sault : Les cahiers de la photographie de Saint-Benoît-du-Sault.

Chéroux, Cl. 2013 Vernaculaires. Essais d'histoire de la photographie. Cherbourg Octeville : le Point du jour.

- 2005 « Portraits en pied... de nez. L'introduction du modèle récréatif dans la photographie foraine », Études photographiques $16: 88-107$.

- 2003 Fautographie. Petite histoire de l'erreur photographique. Crisnée : Yellow Now.

Cuttriss, F. 1915 Romany Life. Experienced and Observed during Many Years of Friendly Intercourse with the Gypsies. London : Mills \& Boon.

De Freitas, Z. \& Ghnassia P. 2001 Photographes de rue, Street Photographers, Minuteros. Hommage collectif. Mialet : Katar Press.

Eltzroth, E. L. 2008 « Georgia Photographers : The First Generation, 1840-1860 », The Georgia Historical Quarterly 92 (1) : 37-64.

Fieschi, J.-C. 2002 « Nicolas Wickelson, photographe ambulant », Club Niepce Lumière 110 : 10-14.

Fontaine, L. 1993 Histoire du colportage en Europe, XVe-XIXe siècle. Paris : Albin Michel.

Freund, G. 2011 [1936] La Photographie en France au dix-neuvième siècle. Essai de sociologie et

d'esthétique. Paris : C. Bourgois, IMEC.

Gilardi, A. 1976 Storia sociale della fotografia. Milano : Feltrinelli.

Girardin, J. 1909 [1889] Le Commis de M. Bouvat. Paris : Hachette.

Hartley, W. D. dir. 1994 Otto Ping. Photographer of Brown County, Indiana, 1900-1940. Indianapolis : Indiana Historical Society.

Hucq-Lajolie, F. S. dir. 1983 Léopold Dussouchet, 1887-1963, photographe ambulant, Juignac. Magnac : Le Montreur d'images.

Jézéquel, H. 1995 « La Photographie dans la fête foraine », Ethnologie française 25(4) : 687-695.

Jowanowitch, M. 1903 « Lettre de Serbie », L'Information photographique 2 : 277-278.

Kailbourn, T. R. \& Palmquist P. E. 2000. Pioneer Photographers of the Far West. A Biographical Dictionary, 1840-1865. Stanford : Stanford University Press.

Lacan, E. 1858 « Les Saltimbanques de la photographie », La Lumière 8 (20) : 1.

Lacoste, A. dir. 2010 Felice Beato. A photographer on the Eastern road. Los Angeles : J. Paul Getty Museum. 
Lacroix, D. 2011 « François Gabriel, photographe montmartrois », Le Cartophile 157 : 3-11.

Legentil, E. 8 mars 1904 « Paris ignoré. Le photographe ambulant », La Presse.

Lenman, R. 2005 « Itinerant photographers ", in R. Lenman dir. The Oxford Companion to the Photograph. Oxford : Oxford University Press : 323-324.

Lerebours, N.-M. P. 1840 Excursions daguerriennes. Vues et monuments les plus remarquables du globe. Paris : H. Bossange, Lerebours, Rittner et Goupil.

Linkman, A. E. 1990 « The Itinerant Photographer in Britain 1850-1880 », History of Photography 14 (1) : 49-68.

Löwe, J.-M. 1866 Mémoires d'un photographe ambulant, le roman de la France. Paris : Balitout, Questroy et Cie.

MacDougall, D. 2005 The Corporeal Image. Film, Ethnography, and the Senses. Princeton : Princeton University Press.

Mahadevan, S. 2013 « Archives and Origins : The Material and Vernacular Cultures of Photography in India », Trans-Asia Photography Review 4 (1). [En ligne], URL : http:// hdl.handle.net/2027/spo.7977573.0004.103.

Marcenaro, G. 1995 « Alphonse Bernoud et les photographes ambulants sur la côte ligure : 1839-1870 », Le Monde alpin et rhodanien 2-4 : 161-174.

Maresca, S. 2004 « L'Introduction de la photographie dans la vie quotidienne ", Études photographiques 15 : 61-77.

Martin-Civat, P. 1975 « Petits métiers du vieux Cognac et du Cognaçais », Revue de recherches ethnographiques. Le Subiet 9(1) : 30-34.

Maurice, P. 2008 «Itinerant photography », in J. Hannavy dir. Encyclopedia of Nineteenth-Century Photography, vol. 1. New York : Routledge : 759-761.

- 1995 « Snippets of History: The Tintype and Prairie Canada », Material History Review 41 : 39-56.

Morand, S. 2001 « Schneider Trudpert », in Dictionnaire mondial de la photographie. Paris : Larousse : 522.

Mullins, C. 2012 The World on a Plate : The Impact of Photography on Travel Imagery and its Dissemination in Britain, 1839-1888. Brighton : University of Sussex.

Nadernhy, M. 17 juin 1893 « Le Photographe ambulant », Le Monde illustré.

Neal, A. \& Parker A. 1982 Los Ambulantes. The Itinerant Photographers of Guatemala. Cambridge : The MIT Press.

Nimis, É. 2005 Photographes d'Afrique de l'Ouest. L'expérience yoruba. Paris : Karthala.

Normandy, G. 1931 « Alfred Le Petit (1841-1909) », L’Esprit français 3(61) : 17-22.

Osborne, P. D. 2000 Traveling Light. Photography, Travel and Visual Culture. Manchester : Manchester University Press.

Papinot, C. 1996 « La Construction sociale de la pose photographique à Madagascar ", L'Ethnographie 92(2) : 195-218.

- 1987 « Profession : photographe de rue à Tananarive (Madagascar) ", Sociétés. Revue des sciences humaines et sociales 15 : 28-30. 
Pinguet, C. 2014 Felice Beato, 1832-1909. Aux origines de la photographie de guerre. Paris : CNRS Éditions.

Potvin, G. 1987 « La Vie chaotique d'un caricaturiste célèbre : Alfred Le Petit », Gavroche 31 : 11-17.

Rosental, P.-A. 1999 Les Sentiers invisibles. Espaces, familles et migrations dans la France du $19^{e}$ siècle. Paris : Éditions de l'École des hautes études en sciences sociales.

Rutherford, E. 2009 Silhouettes. Paris : Citadelles \& Mazenod.

Spude, R. L. 1989 « Shadow Catcher. A Portrait of Arizona's Pioneer Photographers, 1863-1893 », The Journal of Arizona History 30(3) : 233-250.

Steen, U. 1993 " Spurensuche nach frühen Kieler Fotografen. Gregor Renard und seine Brüder », in H. Behling dir. Kieler Stadt- und Marinebilder. 150 Jahre Foto-Atelier Renard. Neumünster : Karl Wachholtz : 37-55.

- 1987 « Die Anfänge der Photographie in Schleswig-Holstein, 1839-1848 », Nordelbingen 56 : 101-150.

Trock 1883 « Le Photographe nomade », La Caricature $159: 11$.

Vidal, L. 1885 Manuel du touriste photographe, 2 vol. Paris : Gauthier-Villars.

Viditz-Ward, V. 1987 « Photography in Sierra Leone, 1850-1918 ", Journal of the International African Institute 57(4) : 510-518.

Wagniart, J.-F. 1999 Le Vagabond à la fin du XIX siècle. Paris : Belin.

Wäspe, R. 1985 Johann Baptist Isenring, 1796-1860 : Druckgraphik. St. Gallen : Kommissionsverlag, Buchhandlung am Rösslitor.

Werner, J.-F. 1997 « Les Tribulations d'un photographe africain », Autrepart 1 : 129-150.

- 1996 « Produire des images en Afrique : l'exemple des photographes de studio », Cahiers

d'études africaines 36(141-142) : 81-112.

\section{NOTES}

1. Cette étude s'appuie notamment sur l'analyse de plusieurs collections du département des Estampes et de la photographie de la Bibliothèque nationale de France (Paris): AD 1112-1 à 4 : Caricatures sur la photographie; AD 7161 : Recueil. Cartes postales fantaisies et publicitaires sur la photographie.

\section{RÉSUMÉS}

Cet article examine la pratique professionnelle et la vie quotidienne des photographes ambulants dans une aire géographique centrée sur la France et élargie à d'autres aires de comparaison en Europe, entre les années 1880 et les années 1930. L'enquête s'appuie sur l'étude des témoignages écrits et graphiques du métier de photographe ambulant et sur plusieurs collections issues 
d'archives publiques (BNF, Archives départementales) et de collections privées. Partant du rôle de cette activité itinérante dans la diffusion massive des pratiques de l'image photographique hors des zones d'activité des studios installés, dans les petites et moyennes villes et dans les campagnes, l'article s'intéresse à la figure sociale du photographe ambulant et analyse les conditions matérielles et quotidiennes de son métier : en restituant les logiques économiques précaires ainsi que les relations entre l'offre commerciale et la demande sociale, se dégage le portrait d'un artisan de la photographie, à la fois praticien expert, artiste fantasque, migrant de passage et inventeur des formes visuelles du portrait qui ont influencé l'histoire des principales pratiques visuelles vernaculaires.

This article examines the professional practice and the daily life of the itinerant photographers in a geographical area centered on France and extended to other comparative areas in Europe, in a chronological arc between the 1880s and the 1930s. The investigation is based on the study of written and graphic testimony on itinerant photographers and on several collections from public archives (BNF, archives départementales) and private collections. The article underlines the role of this itinerant activity in the social diffusion of the photographic image outside the commercial areas of installed studios, in small and medium-sized cities and in the countryside. The analyse focuses on the social figure of the itinerant photographer and scrutinises the material and daily conditions of this activity : in restoring the precarious economic logics and the relations between commercial supply and social demand, emerges a portrait of a photographic craftsman, an expert practitioner, a whimsical artist, a migrant and an inventor of portraiture visual forms that influenced the history of the main vernacular visual practices.

\section{INDEX}

Keywords : Photography, Hawking, Peddling, Wandering, Craftsmanship, Images

Mots-clés : Photographie, Colportage, Commerce ambulant, Vagabondage, Artisanat, Images

\section{AUTEUR}

\section{ILSEN ABOUT}

Centre Georg Simmel, EHESS/CNRS 